\title{
Pricing strategies for emerging markets
}

Emerging markets are expected to contribute to more than $20 \%$ of global sales for prescription drugs and nearly $50 \%$ of industry growth by 2013. However, identifying the appropriate pricing strategy is a key challenge, given that the cost of a therapy in established markets may often exceed the annual income of a typical patient in emerging markets. At present, various options are being explored without a clear framework, thus potentially sacrificing value and overlooking opportunities. We have investigated multiple pricing strategies to create a decision framework when launching a new branded therapeutic in a given emerging market. The potential application of this framework, which is shown in FIG. 1, is highlighted below.

\section{Examples of pricing strategies}

Donation for Gleevec. Imatinib (Gleevec;

Novartis) has transformed the treatment of chronic myeloid leukaemia (CML). However, when it was introduced in India in 2002, it was targeted by local manufacturers producing inexpensive generic versions. Rather than simply withdrawing from India, Novartis worked with the Max Foundation to establish the Gleevec International Patient Assistance Program (GIPAP), through which over $99 \%$ of Indian patients receive imatinib at no cost, undercutting any profit for local manufacturers. Novartis was also able to establish a presence in India, educate physicians and patients about CML and prepare the market for next-generation $\mathrm{CML}$ products such as

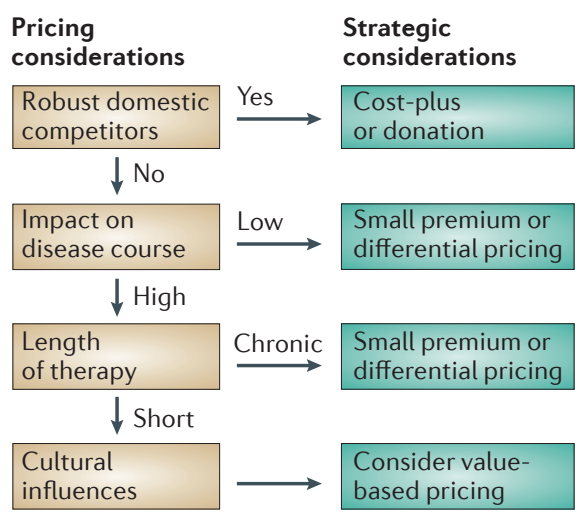

Figure 1 | Pricing decision framework.

Considering the local competition, the impact on disease, the duration of treatment and unique cultural factors in series could help with the identification of more effective pricing strategies. nilotinib. In addition, programmes such as GIPAP may allow companies to donate a product to a non-profit partner and receive tax breaks for the revenue of the product. In other markets with less robust domestic competition and a higher median income (such as Latin America and China), GIPAP works with governments to share the cost of treatments, thus highlighting that the pricing strategy should also reflect unique domestic cultural influences. Alternatively, companies can implement 'cost-plus' pricing with a very small fixed margin added to production costs.

\section{Tiered pricing for recombinant blood} replacement products. Recombinant blood replacement products - such as Pfizer's Xyntha, Bayer's Kogenate and Baxter's Recombinate - reduce the risk of bleeding and infection in patients with haemophilia. The high medical need and chronic nature of these treatments prohibits a price cap. Companies have instead adopted differential pricing strategies by geography, which they have shown can allow some premium pricing.

Price caps for Nexavar. In July 2008, sorafenib (Nexavar; Bayer) became the first drug to be approved for treating unresectable or metastatic hepatocellular carcinoma (HCC) in China. Nexavar offered a robust benefit for these $\mathrm{HCC}$ patients, extending the median time to disease progression by $\sim 2.8$ months. Bayer pursued premium pricing ( US $\$ 7,000$ per month), with the overall cost to the patient capped at 3 months of use, allowing Bayer to maintain a price per dose that was consistent with developed markets while providing a similar level of care. With no robust competitors available for Nexavar, patients are willing to pay for the improved clinical benefit with the assurance of the price cap. The success of this strategy would have been challenged, however, if local competition could undercut the price, or if patients were likely to stay on the therapy for considerably longer than 3 months.

\footnotetext{
Neil Lineberry, Eric Snyder and Seema Gunda are at Leerink Swann, One Federal Street, Boston, Massachusetts 02110, USA.

e-mails: neil.lineberry@leerink.com; eric.snyder@ leerink.com; seema.gunda@leerink.com

The authors declare competing financial interests: see Web version for details.
} 